lateral electrocardiogram lead. There was minimal output through the chest tubes, but because the patient was unresponsive to inotropes, the chest was opened. The patient was resuscitated with open massage. After several minutes of open massage and intracardiac epinephrine, the patient had normal blood pressure and no electrocardiogram changes. His cardiac output was more than $5 \mathrm{~L}$ without inotropic support. There was no bleeding from the suture lines.

After reading this communication, my suspicion is that the BioGlue application to the anterior part of the aortic root suture line migrated through the suture line and embolized the coronary arteries after the patient returned to the intensive care unit. Other possibilities include air or tissue embolus or localized redissection in the proximal root.

I would recommend the use of BioGlue on suture lines only to achieve hemostasis and would use only a minimal amount of the adhesive.

Frank A. Baciewicz, Jr, MD Department of Cardiothoracic Surgery Wayne State University School of Medicine Detroit, Mich doi:10.1016/j.jtcvs.2005.09.013

\section{Right axillary incision: Is it really superior to anterolateral thoracotomy?}

To the Editor:

I read the article by Prêtre and associates ${ }^{1}$ on right axillary incision performed for a wide range of congenital cardiac defects. The authors have described the cosmetic and functional advantage of this approach in various surgical procedures. Although I commend them for using this in a wide array of congenital heart diseases, I was surprised to find that the advantage it allegedly scores over anterolateral thoracotomy has been that there is no interference with breast development. While avoiding the necessity of groin cannulation for establishing $\mathrm{CPB}$, there has not been a single incidence of intraoperative technical complications in a series of 140 patients in whom an anterolateral approach was used for atrial septal defect closure in my previous institution's experience (at G.K.N.M Hospital, Coimbatore, India). ${ }^{2}$ There also has not been any problem with breast de- velopment. The institution of $\mathrm{CPB}$ was conventional in the main incision itself with no significant increase in operative time or subsequent bleeding or hospital stay. Posterolateral thoracotomy may be technically demanding too, and the necessity for groin exposure in small children makes it less attractive than anterolateral thoracotomy. Once the dissection plane is established well in the submammary tissue, an approach via the fourth intercostal space gives good exposure to both the aorta (once thymic tissues are removed) and the venae cavae for cannulation. Both the atria are easily approachable for most congenital defects. When a correct tissue approximation is achieved with no puckering of breast tissue, there is no anticosmesis involved in breast asymmetry nor any cage deformity if well aligned. To stress again, asymmetric breast development is more feared than proven in these patients who undergo anterolateral thoracotomy.

\section{Nagarajan \\ Clinical Fellow in Cardiac Surgery Nottingham City Hospital NHS Trust Nottingham NG5 1PB, United Kingdom}

\section{References}

1. Prêtre R, Kadner A, Dave H, Dodge-Khatami A, Bettex D, Berger F. Right axillary incision: a cosmetically superior approach to repair a wide range of congenital cardiac defects. J Thorac Cardiovasc Surg. 2005;130: 277-81.

2. Muralidharan S, Anup Krishnan V, Varma S K, Nagarajan M. Atrial septal defect closure in young females by anterolateral thoracotomy. Ind J Thorac Cardiovasc Surg. 2004; 20:129-31.

doi:10.1016/j.jtcvs.2005.09.054

\section{Reply to the Editor:}

The comment made by Dr Nagarajan finally comes down to an evaluation of the benefit of thoracic incisions on overall cosmesis and patient acceptance of the incision. His statement that anterolateral thoracotomy does not lead to significant breast deformation is correct only when the incision is applied in adults. In this age group, the breasts are developed and the inframammary fold is delineated. An anterolateral thoracotomy, however, in a prepubescent girl does lead to breast and chest asymmetry. Because full thoracic development requires more than a decade, and because troubles related to the private sphere are difficult to express, the cosmetic and psychological results of the anterolateral thoracotomy have been poorly evaluated and often ill-estimated. The fact that most patients-even the dissatisfied ones-never actively expressed complains regarding the incision (something that appears futile in view of the correction that was performed on the heart) was put to the credit of the approach. Bleiziffer and coworkers ${ }^{1}$ convincingly demonstrated that significant impairment (more than $20 \%$ of volume difference) in the development of the right breast occurred in $61 \%$ of the female patients after an anterolateral thoracotomy, yet only a minority of them expressed their discontentment. The asymmetry was the consequence of damage not only to the breast gland but also to the pectoralis muscles (which need be disinserted from the rib) and sometimes to the rib and costochondral junction. This also explains the suboptimal results achieved in boys. Interestingly, in this study, women with anterolateral thoracotomy and asymmetrical breasts had a better psychological acceptance of their image than those with a classic sternotomy and symmetrical breasts, evidencing the burden played by the visibility of the incision. Even if the subxiphoid approach, which leaves the upper part or "décolleté" of the thorax free, will be better accepted than a full sternotomy, the visibility of the incision will remain a nuisance for some young people. ${ }^{2}$ The posterolateral approach requires division of the latissimus dorsi and sometimes part of the serratus anterior muscle. ${ }^{3}$ The cosmetic appearance of the scar is certainly comparable with our axillary incision. Stature problems and weakness of the shoulder are, however, possible consequences of this incision. In our opinion, the axillary incision, because it is located in a muscle-free area and because it spreads more than it divides muscles, leads to yet incomparable cosmetic and functional results in children. Schreiber and coworkers ${ }^{4}$ recently reported similar results with the same incision (using a central cannulation) in an older population. To us, the anterolateral incision as advocated by Nagajaran is indicated in adults only, especially if the cardiac repair can be performed endoscopically. $^{5}$ 